\title{
Exploring the Understandability of a Hybrid Process Design Artifact Based on DCR Graphs
}

\author{
Abbad Andaloussi, Amine; Burattin, Andrea; Slaats, Tijs; Petersen, Anette Chelina Møller; Hildebrandt, \\ Thomas; Weber, Barbara
}

Published in:

International Conference on Business Process Modeling, Development and Support International Conference on Evaluation and Modeling Methods for Systems Analysis and Development

Link to article, DOI:

10.1007/978-3-030-20618-5_5

Publication date:

2019

Document Version

Peer reviewed version

Link back to DTU Orbit

Citation (APA):

Abbad Andaloussi, A., Burattin, A., Slaats, T., Petersen, A. C. M., Hildebrandt, T., \& Weber, B. (2019). Exploring the Understandability of a Hybrid Process Design Artifact Based on DCR Graphs. In International Conference on Business Process Modeling, Development and Support International Conference on Evaluation and Modeling Methods for Systems Analysis and Development (pp. 69-84). Springer. Lecture Notes in Business Information Processing Vol. 352 https://doi.org/10.1007/978-3-030-20618-5_5

\section{General rights}

Copyright and moral rights for the publications made accessible in the public portal are retained by the authors and/or other copyright owners and it is a condition of accessing publications that users recognise and abide by the legal requirements associated with these rights.

- Users may download and print one copy of any publication from the public portal for the purpose of private study or research.

- You may not further distribute the material or use it for any profit-making activity or commercial gain

- You may freely distribute the URL identifying the publication in the public portal 


\title{
Exploring the Understandability of a Hybrid Process Design Artifact Based on DCR Graphs
}

\author{
Amine Abbad Andaloussi ${ }^{1}$, Andrea Burattin ${ }^{1}$, Tijs Slaats ${ }^{2}$, Anette Chelina \\ Møller Petersen ${ }^{3}$, Thomas T. Hildebrandt ${ }^{2}$, and Barbara Weber ${ }^{1,4}$ * \\ 1 Software and Process Engineering, Technical University of Denmark \\ 2800 Kgs. Lyngby, Denmark - amab@dtu.dk \\ 2 Department of Computer Science, University of Copenhagen \\ 2100 København $\varnothing$, Denmark \\ 3 Business IT Department, IT University of Copenhagen \\ 2300 København S, Denmark \\ 4 Institue of Computer Science, University of St. Gallen, \\ 9000 St. Gallen, Switzerland
}

\begin{abstract}
Process design artifacts (e.g., process models, textual process descriptions and simulations) are increasingly used to provide input for requirements elicitation and to facilitate the design of business processes. To support the understandability of process models and make them accessible for end-users with different backgrounds, several hybrid representations combining different design artifacts have been proposed in the literature. This paper investigates the understandability of DCRHR, a new hybrid process design artifact based on DCR graphs. Using eye-tracking and think-aloud techniques, this paper explores the benefits and challenges associated with the use of different design artifacts and investigates the way end-users engage with them. The results motivate the use of DCR-HR and provide insights about the support it provides to end-users with different backgrounds.
\end{abstract}

\section{Introduction}

In the development of today's Process-Aware Information Systems (PAIS), process design artifacts (shortly process artifacts) play a central role both in the enactment and the management of business processes. Besides providing a blueprint for process execution and enabling simulation and model checking, process artifacts provide input for requirements elicitation and allow a shared understanding of the business process [6][p. 66-67]. Depending on the target audience and the nature of the task to be fulfilled, different process artifacts (e.g., process models, textual process descriptions and interactive simulations) can be more or less beneficial [18]. The background of the process stakeholders (i.e., domain experts and IT specialists) influences the degree of formality required

\footnotetext{
* Work supported by the Innovation Fund Denmark project EcoKnow (7050-00034A); the third author additionally by the Danish Council for Independent Research project Hybrid Business Process Management Technologies (DFF-6111-00337).
} 
to describe the business process specifications. Domain experts often lack the skills to derive knowledge from formal process models; thus, they tend to rely on informal process artifacts to describe and communicate their knowledge of the domain. Conversely, IT specialists are more familiar with formal representations; thus they tend to use process models to derive a fine-grained understanding of the business process [13]. As the development of PAIS involves the collaboration of both process stakeholders, the deployment of a single type of process artifact is usually not sufficient to support knowledge transfer and provide an overarching understanding. Moreover, a process artifact can be used in several contexts to fulfill different tasks. For instance, it can be used (a) to check the constraints governing the execution of the business process activities, (b) to extract contextual information about the business process (c) to determine the behavior of a specific process execution based on the case history. Depending on the task, one artifact might be better suited than the others. In addition, the use of a single language to describe the business process can negatively impact its understandability. This is clearly the case with declarative languages, which despite their enhanced flexibility (compared to imperative languages), they are still controversial in terms of understandability especially with regards to novice end-users [7]. This, in turn, suggests the need to support declarative process models with other representations in different languages to improve their understandability.

In order to overcome the limitations of single process artifacts, a set of hybrid process artifacts have been proposed in the literature (e.g., $[4,5,10,14,15,20]$ ). These approaches combine different types of process artifacts and claim an enhanced user understanding of the process model. However, none of these approaches has explored the reading patterns of stakeholders with different backgrounds when dealing with all the previously mentioned tasks. This work reports the results of an exploratory study investigating the understandability of a hybrid process artifact (called "DCR Hybrid Representation" or DCR-HR shortly) combining a declarative DCR (Dynamic Conditional Response) graph model [8] with textual annotations depicting the law and an interactive simulation. The study investigates the usefulness of hybrid process artifacts by (a) identifying the benefits and challenges associated with each of the DCR-HR artifacts, (b) observing the way end-users with different backgrounds engage with the different artifacts proposed by DCR-HR, and (c) exploring the way the different DCRHR artifacts can be used to fulfill different tasks. To support the findings, this work deploys a novel approach to investigate the reading patterns of end-users and uses concepts from grounded theory [3] to extract subjective insights from the participants who took part in the study.

The remaining of this paper is structured as follows. Section 2 provides an overview of hybrid process artifacts and describes the related work. Section 3 presents the research method followed to plan and conduct the exploratory study. Section 4 reports the results of the analysis. Section 5 discusses the analyzed findings and highlights the circumstances when the use of a hybrid process artifact can be beneficial. Finally, Section 6 wraps up the main findings of the study and highlights the directions for future work. 


\section{Background and Related Work}

Hybrid process representations have been introduced in the literature to designate (a) hybrid languages or (b) hybrid process artifacts. Hybrid languages (e.g., [17]) combine existing languages in order to enable a concise and precise representation of business processes. Hybrid process artifacts, in turn, combine two or more process artifacts overlapping in the description of some business process aspects. DCR-HR is a hybrid process artifact combining two static process artifacts (i.e., a declarative process model and a textual description of the process) with an interactive process artifact (i.e., an interactive simulation showing the possible outcomes depending on the user input). DCR-HR (cf. Fig. 1) aims at improving the understandability of DCR process models and helping end-users with different backgrounds to make sense of law's digitized models $[1,2]$.

The literature proposes similar hybrid process artifacts. These representations can be categorized into the following sets: (a) process artifacts combining a process model with textual process descriptions and (b) process artifacts combining a process model with an interactive simulation. In the former set, several approaches (e.g., $[10,14])$ combining an imperative process model in BPMN (Business Process Modeling Notation) ${ }^{5}$ with business rules described textually have emerged. These approaches have been evaluated in [15] where the authors have investigated the effect of using linked rules (i.e., a type of business rules) on the understandability of process models. The findings of the study show that the combination of BPMN and business rules is associated with higher performance and reduced mental effort. Similarly in [4], the authors evaluated the understandability of a hybrid process artifact combining a declarative process model and a textual artifact revealing the hidden dependencies in the model. The results demonstrate a lower response time and reduced mental effort when using the proposed hybrid representation. In the latter set, two approaches combining a process model with an interactive simulation (i.e., $[5,20]$ ) have been proposed. The former approach combines a declare process model with test cases to support the maintainability and modeling of process models. This approach was evaluated in [18] where the authors have analyzed the verbal data transcribed from a set of modeling sessions. The results show that the proposed process artifact helped to increase the perceived model quality. The latter approach (i.e., [5]) proposes a hybrid process artifact combining a process model in DCR with an interactive simulation. A similar representation is used as a basis to derive the hybrid process artifact scrutinized in this work.

The evaluation presented in this work differs from the existing ones in different aspects. In term of experimental subjects (cf. Section 3), unlike the existing evaluations which have mainly covered university students, this study recruits participants with different backgrounds (i.e municipality case-workers and people from academia), which in turn allows evaluating the usefulness of DCR-HR in a wider scope. In term of experimental material (cf. Section 3), while the existing evaluations have usually used made-up process descriptions or domain-

\footnotetext{
${ }^{5}$ See https://www.omg.org/spec/BPMN/2.0/About-BPMN/
} 


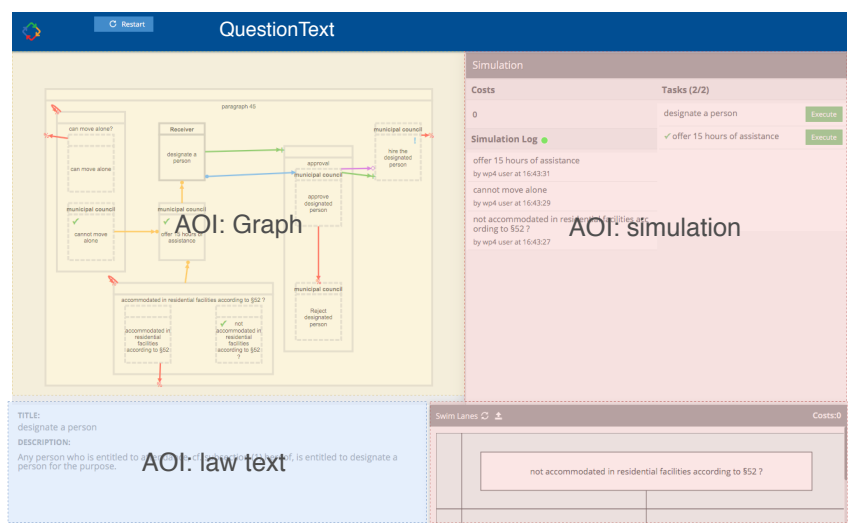

Fig. 1: A view showing the DCR-HR layout. At the analysis, this view is divided into $3 \mathrm{AOIs}$ referring to the different artifacts. A higher resolution of this figure is available at http://andaloussi.org/papers/BPMDS2019/DCRHRLayout.pdf

free process models (where activities are labeled with single characters e.g., A, B ...), this work deploys digitized models of the law meant to be used by caseworkers to handle citizens' requests. In addition, the experimental tasks used in the proposed evaluation are crafted to cover different contexts (i.e., checking constraints, checking contextual information or determining the behavior of a process instance based on a given case history, cf. Section 3). In term of analysis approach (cf. Section 3), this work extends the findings of the existing evaluations by investigating the reading patterns obtained from the eye-tracking gaze recordings and triangulating them with subjective insights obtained from thinkaloud sessions. Hence, the proposed approach provides novel insights into the usefulness of hybrid process artifacts.

\section{Research Method}

This section presents the research method deployed to plan and conduct this exploratory study. The following paragraphs introduce the research questions addressed in this work, highlight the key design aspects and provide insights about the measurements used during the analysis.

Research Questions This work considers three types of process artifacts (i.e., process models, textual process descriptions, and interactive simulations) which are commonly used to represent business processes. In order to support end-users when dealing with business process representations, it is necessary to understand the benefits and challenges associated with their use. The first research question is formulated as follows: RQ1: What are the benefits and challenges associated with each of the artifacts proposed by DCR-HR?

Process artifacts are used by domain experts and IT specialists. The two groups have different backgrounds which raise the question of whether the disparity of backgrounds is reflected in the way they use the different artifacts. The 
second research question is formulated as follows: RQ2: How do end-users engage with the different DCR-HR artifacts?

Finally, as process artifacts can be used to fulfill different tasks, it is also essential to investigate whether a single process artifact or a specific combination of process artifacts can provide global support in solving different types of tasks. The third research question is formulated as follows: RQ3: How are the different DCR-HR artifacts used to fulfill different task types?)

Participants To investigate the above research questions, an exploratory study is conducted. In this study data is collected from 5 case-workers from Syddjurs municipality in Denmark and 10 academics (i.e., students, faculty) from the Technical University of Denmark and the IT University of Copenhagen. Caseworkers serve as proxies for domain experts. They have proficiency in reading law texts but lack knowledge in process modeling. Academics serve as proxies for IT specialists. They have knowledge in process modeling, but lack proficiency in law.

Material As part of this exploratory study, participants had to perform several model comprehension tasks. The process deployed for this study originates from Section $\S 45$ of the "Consolidation Act on Social Services" ${ }^{\text {. The material }}$ was provided in both English and Danish depending on the participant's preference. The designed artifacts are intended to be complementary to each other. Nevertheless, a considerable overlap exists between all of them. Following the modeling of an expert in DCR graphs, the graph captures the requirements of the law text, while the simulation represents a concrete implementation of the DCR graph. The experiment comprises a familiarization and 6 tasks. It is possible to categorize these tasks into constraint tasks, decision tasks and scenario tasks. Constraint tasks comprise questions about the relationships between pairs of activities in the process model. These questions reflect the circumstance where an end-user has to maintain a process model (e.g., update a constraint between two activities). In this context, it is necessary for the end-user to identify the specific constraint that should be changed in the model. Decision tasks comprise questions where the system is prompting the end-user to decide among several options. In that regard, the end-user should be able to identify the contextual information required to guide her/his decision-making process in order to achieve the desired outcome. Finally, scenario tasks illustrate the follow-up on customer cases. Namely, this type of tasks provide the end-user with a case history and ask her/him to determine the allowed behavior based on the given history. For each of these types, 2 tasks were designed. The experiment material can be found online at http://andaloussi.org/papers/BPMDS2019/Material.pdf.

Procedure Prior to the experiment, the participants were given a screening form to check their physical ability to participate in an eye-tracking experiment, afterward, they were given a pre-experiment questionnaire to collect their background information. At the beginning of the experiment, each participant

\footnotetext{
${ }^{6}$ http://english.sm.dk/media/14900/consolidation-act-on-social-services.pdf (Eng), https:// www.retsinformation.dk/Forms/R0710.aspx?id=197036 (Dan)
} 
has received an introduction to DCR-HR where the semantics of the different DCR relations have been presented and the features of the DCR platform have been demonstrated. During the eye-tracking experiment, the 6 understandability tasks were sequentially displayed. At the end, a retrospective think-aloud session [9][p. 104-108] was held to collect insights about the use of DCR-HR.

Settings The experiment material was designed and presented in the DCR platform ${ }^{7}$. A view showing the presented layout in depicted in Figure 1. Prior to each eye-tracking session, a calibration procedure was conducted to ensure a good data quality. The gaze data were collected using Tobii X3-120 ${ }^{8}$. Fixation data [9] [p. 22]) were derived using the I-VT Algorithm [11] in Tobii Pro Studio 3.4.8. Finally, all the subjective insights provided verbally by the participants were recorded with their consent.

Analysis Approach To answer our research questions, two different types of analysis are proposed. Namely, we use concepts from grounded theory [3] to extract subjective insights from think-aloud data and process mining techniques to explore the participants' reading patterns through attention maps. The subjective insights are extracted with the support of Atlas.ti ${ }^{9}$. During this process, the most reoccurring aspects related to the use of the different DCR-HR artifacts are identified and then grouped into categories based on their common traits.

The attention maps are obtained from the fixation data provided by the eye-tracking software. After dividing the stimulus into three areas of interest (AOIs) [9] [p. 187-230] (each referring to a distinct DCR-HR artifact, cf. Figure 1), a time-stamped fixation data-set comprising a set of scan-paths (i.e., distinct sequences of fixations illustrating the reading paths participants) is exported and transformed into an XES event $\log ^{10}$. After identifying the direct relationships in the $\log [16]$, a descriptive process model (referred as attention map in the context of this work) illustrating the participants' reading pattern is generated. Examples of such attention maps are shown in Figures 2 and 3. The different AOIs are represented as activities in the attention maps, while the transitions between the different AOIs are represented as edges. In order to analyze the different reading patterns, the total fixation duration on each AOI (i.e., the sum of the duration of all fixation landing on a particular area of the stimulus [9] [p. 377-386]) and the frequency of transition between each pair of AOIs are extracted from the fixation data-set. Afterward the mean fixation duration $(D)$ and mean transition frequency $(F)$ are derived by dividing each measure by the number of traces (i.e., scan-paths) used to discover the attention map. These two measurements are projected respectively on activities and edges in the attention map to allow comparing the reading patterns in different attention maps.

\footnotetext{
7 see http://dcrgraphs.net/ and http://wiki.dcrgraphs.net/

${ }^{8}$ See https://www . tobiipro.com/product-listing/tobii-pro-x3-120/

9 A qualitative data analysis tool. See https://atlasti.com

10 See http://www.xes-standard.org/start
} 


\section{Findings}

This section reports the finding of the study answering the research questions presented in Section 3. Section 4.1 identifies the benefits and challenges associated with each of the DCR-HR artifacts. Section 4.2 investigates the way end-users with different backgrounds engage with DCR-HR. Section 4.3 scrutinizes the end-users' reading patterns when dealing with different types of tasks.

\subsection{What Are the Benefits and Challenges Associated with Each of the Artifacts Proposed by DCR-HR? (RQ1)}

The individual think-aloud sessions held with the participants after the experiment provide rich insights about the usability of DCR-HR as well as the support provided by the different artifacts. As explained in Section 3, think-aloud audio recordings were transcribed, then following a qualitative coding approach different labels were assigned to distinguish the different aspects emphasized by the participants during the think-aloud sessions. This section investigates the codes associated with the benefits and challenges of the DCR graph, the law text and the simulation.

The results show that the DCR graph helped several participants to get a good overview about the business process (e.g., "The model I mainly used it to identify like how the overall process works"). As each activity in the DCR graph is linked to its corresponding law fragment, the DCR graph allowed the participants to identify and navigate through the different sub-sections of the law (e.g., "You can highlight different sections of law through [the] model"). Some academics mentioned that the DCR graph helped them to understand the interplay between the different process activities (e.g., "I use the model to see [the] interaction between the four different activities"), whereas some case-workers were challenged by the semantics of the DCR relations. These challenges were inferred from their quotes during the think-aloud, as several participants were unable to identify the appropriate DCR relation specifying a certain behavior. In addition, some participants found the DCR graph very abstract and pointed out that the model was sometimes missing the details of the law text (e.g., "If you only have the model it's very abstract", "The strange thing is that many things which the law is talking about the model did not talk about").

The law text, in turn, provided the participant with details which were missing in the DCR graph (e.g., "I mean I guess it provided more details in some cases than the model", "The law text might be able to add some details that can't be in the model ", "If I didn't think that model accurately captured enough for me to answer the question then I would read the whole text instead"). The participants also mentioned that the law text supported their decision-making process when the DCR graph allows for more than one choice (e.g., "When I had to use the law text was for questions about 'should I do this' at all, for example should I give personal permission should I take the accept or should I take the reject button on an activity."). In addition, several case-workers have shown a preference for the law text as they were already familiar with reading and interpreting 
law paragraphs (e.g., "I mostly used the law text because that's what I'm used to looking at"11). In turn, many of the academics had difficulties to understand the legal terms and the linguistic patterns used in the law text (e.g., "I think understanding this law jargon was kind of difficult", "I tried to read the law text to understand the law but it actually didn't help at all because the language that is used is pretty formal"). Therefore, some academics were avoiding referring to the law text to extract knowledge about the business process (e.g., "It is not so easy to read the law text ... I have totally ignore it").

Finally, the interactive simulation, allowed the participants to check the viability of different process executions (e.g., "The simulation is helpful to see the possible paths", "You can actually see if you have a viable execution"). Moreover, some academics have affirmed that using the simulator helped to reduce the mental effort required to keep track of the dependencies between the different DCR relations (e.g., "It's a little much to have all the steps in your mind while you're going ...", "It is easier to see it simulated instead of manually analyze the model"). These comments fall in line with the previous claims about the role of interactive simulations in improving the understandability of declarative process models [19]. The analysis of the transcripts shows also that the simulation helped participants to validate the insights retrieved from the DCR graph (e.g., "You can like simulate the process then you like get a clear understanding of how the process works ... if you're in doubt of like relations or anything in the graph then you can use the simulation to like confirm what you actually think about the model "], ". . checking if it is exactly what I thought the model is doing it's actually doing it"). Yet, other participants have pointed out some drawbacks associated with the use of the simulation. In particular, some academics thought it was inefficient to restart the simulation all the way back at the beginning every time an undesired state is reached (e.g., "Actually this was not very convenient because you click the all way through and if you miss click, which I actually did, you need to do it again"). In addition, some academics have abstained from using the simulation because they were able to mentally simulate the process (e.g., "Primarily, I didn't use the simulator at all because I pretty much simulated in my head"). Other case-workers were not used to such an approach in their work practice and thought that the use of the simulation could be time-consuming for them (e.g., "I'm used to working under very high work pressure, so getting in and checking such things through that way is not in my habits"11, "You would spend too long to press and read all four options, then press again and read three new options, then press again and there will be five new options"11).

These insights show that each artifact has some strengths - but has also some weaknesses. Although the participants have been exposed to the three process artifacts during all the experiment trials, it can be seen that they have shown a preference for different process artifacts based on their perceived usefulness and the context in which they have been deployed. In the meantime, the participants have also reported a set of challenges they faced when interacting with these artifacts. This suggests that no single artifact can be enough to provide an

\footnotetext{
${ }^{11}$ Quote translated from Danish
} 
overarching understanding of the business process for end-users with different backgrounds, which supports the idea of combining all these artifacts into a hybrid representation to complement each other and make up for their individual weaknesses.

\subsection{How do End-users Engage with the Different Artifacts Proposed by DCR-HR? (RQ2)}

This section investigates the reading patterns of case-workers and academics in order to obtain more insights about their use of DCR-HR. We differentiate in our analysis between case-workers and academics because of their different backgrounds. As mentioned in Section 3, case-workers have proficiency in reading law texts, but lack knowledge in process modeling. Academics, in turn, have a background in process modeling, but lack proficiency in reading law texts. Based on their different backgrounds, we expect that case-workers and academics might use different artifacts (in line with their background) to answer the given tasks. We use attention maps and the insights obtained from think-aloud to explore this assumption. Hereby, activities represent the different artifacts and the mean dwell time measure and the corresponding proportions show the distribution of attention between the different artifacts, while the mean transition frequency and the corresponding proportions provide insights about the interactions between the different artifacts (cf. Section 3).

Figures $2 \mathrm{a}$ and $2 \mathrm{~b}$ show the attention maps comparing the reading patterns of case-workers and academics when answering the given tasks. These visualizations show that both groups typically started by reading the DCR graph, which is reasonable since the DCR graph is placed in the center of the screen and occupies a large portion of it (cf. Figure 1). Furthermore, it can be observed that academics spent substantially more time looking at the different artifacts compared to case-workers (cf. mean fixation duration $D$ in Figures $2 \mathrm{a}$ and $2 \mathrm{~b}$ ). This observation is supported by the subjective insights obtained from the transcripts. Indeed case-workers have affirmed using sometimes common sense or relating to knowledge acquired through experience when answering to some tasks (e.g., "If the recipient is unsatisfied, then, of course, you can change the decision [while the DCR graph shows clearly that such a decision cannot be reversed]"11). However, academics, when asked whether they have used common sense, they all affirmed restricting themselves only to the provided artifacts.

The attention map depicted in Figure 2a indicates that case-workers have split their attention mainly between the DCR graph and the law text. This is also supported by the think-aloud data where the majority of case-workers pointed out that they did not use the simulator, but only the graph and the law text (e.g., "I have either read through the law text or the model but I have not used the simulation."11). Other case-workers combined all the three artifacts when solving the given tasks. In particular, a case-worker affirmed to rely on the law text but still using the simulation as a means for validation, while another case-worker mentioned using the simulation but only twice during the experiment. These insights line up also with the proportions of transitions between the artifacts. 


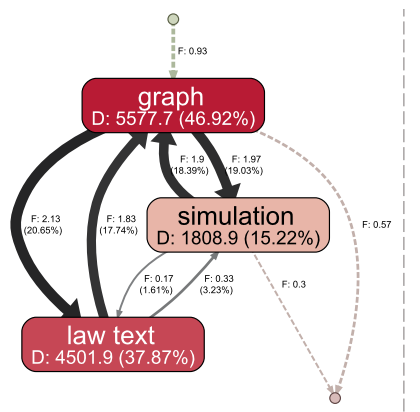

(a) Case-workers

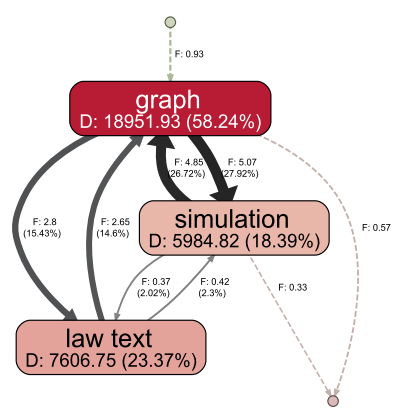

(b) Academics

Fig. 2: Attention maps comparing the reading patterns of case-workers and academics. $D$ refers to the mean fixation duration and $F$ refers to the mean transition frequency between two AOIs (cf. Section 3).

Indeed as shown in Figure 2a, case-workers did roughly the same number of transitions between the graph and the simulation and between the graph and the law text. This suggests that case-workers have generally interacted with all the different artifacts when answering the given tasks.

When looking at the attention map of academics in Figure 2b, a different reading pattern can be observed. Indeed, the distribution of attention shows that academics gave most attention to the DCR graph and split the rest of their attention between the law text and the simulation. As mentioned in Section 4.1 academics were challenged by the legal terms and the linguistic patterns used in the law text which can be a possible explanation for the limited attention on the law text (compared to case-workers). The proportion of transitions between the different artifacts show that academics did almost twice more transitions between the graph and the simulation than between the graph and the law text. These insights show that academics have not only spent a limited time on the law text but also switched less often between the DCR graph and the law text. In addition, one can argue that the academics interacted more with the DCR graph and the simulation than with the law text, which can be justified when looking at the insights obtained from the think-aloud (e.g., "I could actually solve the questions by not looking at the law but the model and the simulator").

Overall, the attention maps (cf. Figure 2) suggest that end-users with different backgrounds use different artifacts to understand the model. Hereby, the hybrid nature of DCR-HR can provide a unified representation that can make process models accessible for end-users with different backgrounds.

\subsection{How Are the Different DCR-HR Artifacts Used to Fulfill Different Task Types? (RQ3)}

This section investigates the way end-users change their reading patterns when dealing with different tasks. As explained in Section 3, the participants were given different types of tasks (i.e., constraint, decision and scenario tasks) 
to illustrate the different contexts where process artifacts are used in real-life. On that matter, it is assumed that each task type would be efficiently accomplished using a certain combination of artifacts (cf. Section 3). To explore this idea, attention maps are used to scrutinize the attention distribution and transition patterns of the participants.

Figures $3 \mathrm{a}, 3 \mathrm{~b}$ and $3 \mathrm{c}$ depicts the attention maps associated with the reading patterns of participants solving constraint, decision and scenario tasks respectively. These visualizations reveal a different reading pattern for each type of task. Figure 3a shows that in constraint tasks (i.e., questions about the relationships between pairs of activities in the process model, cf. Section 3), the participants gave most attention to the DCR graph, and split the rest of their attention between the simulation and the law text. This pattern can be explained by the nature of constraint tasks and the subjective insights obtained from the think-aloud. In fact, several participants highlighted the ability of the DCR graph to show the interplay between the different process activities (cf. Section 4.1). However, the think-aloud shows also that some other participants were challenged by the semantics of the DCR relations and were constantly using the simulation to clarify the implications of the different relations on the model behavior (e.g., "The simulator, I used when I was in doubt because, the different arrows I wasn't always sure what they did, so then I rendered simulator ...then you could actually know for sure if you could do this after this or not"). These subjective insights can be also seen from the high number of transitions between the DCR graph and the simulation. Indeed, the participants did twice more transitions between these two artifacts compared to between the graph and the law text.

When looking at decision questions (i.e., questions about contextual information intended to guide the decision-making process of the end-user, cf. Section 3) a different reading pattern can be observed (cf. Figure 3b). Indeed, the participants split their attention mainly between the DCR graph and the law text. This pattern suggests that, when asked to choose among several options (cf. Section 3), participants have referred to the law text to support their decisionmaking process. Nevertheless, as mentioned in Section 4.1, the DCR graph was also used by the participants to navigate between the different law fragments. This subjective insight explains also the high number of transitions between the graph and the law text in decision tasks.

The reading pattern in scenario tasks (i.e., questions about the allowed model behavior based on a given history, cf. Section 3) differ from both constraint and decision tasks (cf. Figure 3c). First, it is clear that the participants spent relatively less time on the law text while switching their attention mainly between the DCR graph and the simulation. This observation is inferred from both the distribution of attention between the different artifacts and the transition patterns. Hereby, one can argue that the participants have mainly combined the DCR graph and the simulation to answer scenario tasks. This assumption is supported by the think-aloud data where participants affirmed combining the DCR graph and the simulation when given questions comprising a sequence 


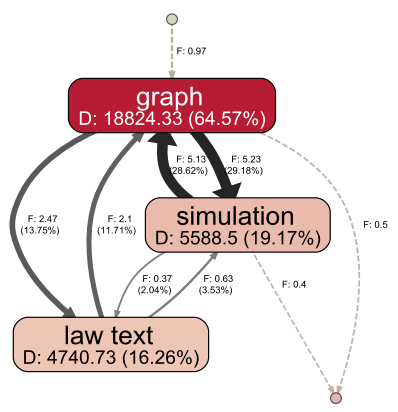

(a) Constraint tasks

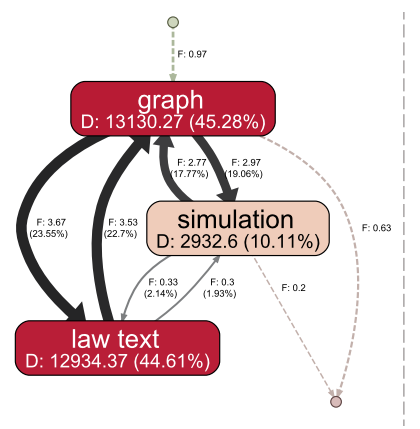

(b) Decision tasks

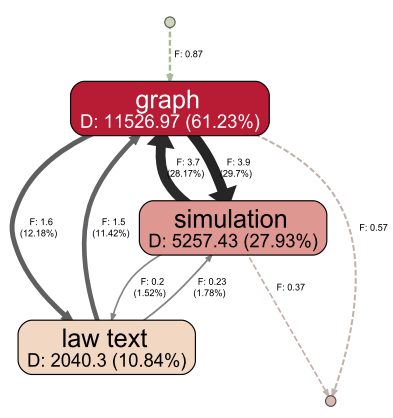

(c) Scenario tasks

Fig. 3: Attention maps comparing the reading patterns of the participants when dealing with different types of tasks. $D$ refers to the mean fixation duration and $F$ refers to the mean transition frequency between two AOIs (cf. Section 3).

of activities and being asked to determine the model behavior from the set of next enabled activities (e.g., "When the question is in a scenario then I use the simulator, because it's easy to see what happens after").

The reading patterns in constraint, decision and scenario tasks share one common trait that is the limited number of transitions between the law text and the simulation. The think-aloud data support this trait as the participants could not see any circumstance where the combination of law text and simulation is beneficial (e.g., "Simulation and law text doesn't go well together because that if you can actually solve it with the simulator, you don't need the law text").

Overall, the insights provided by the attention maps show that the participants combined different artifacts when answering different tasks, which was manifested in the different reading patterns depicted in Figure 3. On those grounds, we suggest that different type of tasks require combining different artifacts. Therefore, the deployment of hybrid process artifacts such as DCR-HR can support end-users when dealing with different tasks in a situation-specific manner.

\section{Discussion}

The disparity of the participants' background and the diversity of the given tasks provide important insights about the understandability of DCR-HR. The analysis of the transcripts revealed that each artifact is associated with a set of benefits and challenges. As presented in Section 4.1, the DCR graph allowed the participants to familiarize with the process scope and provided insights about the constraints governing the execution of the different process activities. Yet, some participants were challenged by the semantics of the different DCR relations. The interactive simulation proposed as part of DCR-HR can help to complement the understanding of the DCR graph by allowing end-users to offload their memory and gradually track the dependencies between the different constraints. 
Nonetheless, its usage was still inefficient and time consuming for some participants. The law text, in turn, was used to complement the information of the DCR graph and provided support in the participants' decision-making process. However, most academics were challenged by reading the law. These insights suggest that a single artifact may not be capable of providing a clear and overarching understanding of the business process. Alternatively, the deployment of a hybrid representation (e.g., DCR-HR) can help to make up for the weakness of individual artifacts. This idea is also supported by the dual coding theory [12] which motivates the combination of graphical and textual artifacts to covey information more effectively.

The reading patterns explored in Section 4.2 come to support the subjective insights provided by the participants. Indeed, case-workers and academics showed different reading patterns when interacting with DCR-HR. Although the majority of case-workers relied on the law text to answer the given tasks, they have additionally been guided by the DCR activities and some of them used the simulation. Academics, in turn, were highly challenged by the law and interacted mainly with the DCR graph and the simulation. This difference of reading patterns can be associated with the disparity of the background of caseworkers and academics, which also reflects the circumstance where both domain experts (represented by case-workers) and IT specialists (represented by academics) are challenged when being exposed to unfamiliar process artifacts [13]. The deployment of a hybrid process artifact (e.g., DCR graph) in turn, will help to overcome this issue by providing a hybrid representation that is understandable to both stakeholders. Moreover, several authors in the literature have linked the understandability of the business process with the effectiveness of communication between the different stakeholders [6][p. 104-108] and [7,13]. As a good understanding of the process would help to clarify the terms and relationships in the domain and prevent miss-interpretations of the process, the deployment of a hybrid process artifact is also expected to foster the communication during the development of PAIS.

The differentiation between different tasks has been clearly reflected in the end-users' reading patterns. The attention maps and the think-aloud output analyzed in Section 4.3 show that the participants have adapted their reading pattern in line with the different task types they have been given. Indeed, the participants gave most attention to the DCR graph in constraint tasks and used the support of the simulation to clarify the information extracted from the DCR graph, whereas in decision tasks the participants have switched their attention to the law text and used the DCR graph as a navigation tool to identify the law fragment in question. In scenario tasks, the participants have combined the simulation and the DCR graph to infer the model behavior at different stages of execution. These indications raise another aspect to be taken into consideration when offering tool support to end-users. Indeed besides the disparity of stakeholders' backgrounds, different types of tasks may require the use of different combination of artifacts. 
The outcome of this study is twofold. On the one hand, it motives the deployment of hybrid process artifacts and emphasizes the importance of enriching process models with textual artifacts and tool supports (e.g., interactive simulations). On the other hand, as the majority of hybrid process artifacts proposed in the literature combine either a textual artifact or an interactive simulation with a process model (cf. Section 2), this work gives empirical insights allowing to enhance the support offered by existing approaches. In this context, we encourage customizing the tool support based on the background of end-users and the nature of the tasks at hand. This support can, for instance, be implemented by learning from the end-users' behavior and context of use in order to bring the artifact with the most value to their attention, while at the same time ensuring that the end-users can freely switch between the different artifacts.

Finally, it has to be noted that the outcome of this study is subject to limitations with regards to the limited number of participants and the exploratory nature of the study. In this sense, it is difficult to generalize the reported findings. Nevertheless, the results provide good insights about the understandability of DCR-HR and can serve a basis for the upcoming studies.

\section{Conclusion and Future Work}

This work summarizes the findings of an exploratory study investigating the understandability of DCR-HR. The results of the analysis suggest that (a) no single artifact is capable of providing a clear understanding of the process, (b) participants with different backgrounds use different combinations of artifacts, (c) different task types require combining different artifacts. All these findings motivate the use of DCR-HR and support its ability to help end-users with different backgrounds to perform different types of tasks.

As future work, we consider the following: (1) First and foremost, we are planning to conduct a follow-up study to further investigate and validate the insights reported in this work. Following a controlled experimental design, we will deploy a control group and treatment group to compare the understandability of process models with and without the support of a hybrid representation. (2) Secondly, we are planning to link the understandability of DCR-HR with the performance of the participants. To this end, the performance of the control and treatment groups will be analyzed and compared when dealing with different types of tasks. (3) Finally, with the availability of more data, we are planning to explore the machine learning capabilities to predict end-users' performance based on the gathered behavioral features. This, in turn, will empower end-users by adapting at run-time the support provided by DCR-HR.

\section{References}

1. A. Abbad Andaloussi, T. Slaats, A. Burattin, T. T. Hildebrandt, and B. Weber. Evaluating the understandability of hybrid process model representations using eye tracking: First insights. In Business Process Management Workshops, pages 475-481. Springer International Publishing, Cham, 2019. 
2. A. A. Andaloussi, T. Slaats, A. Burattin, T. T. Hildebrandt, and B. Weber. A Research Model to Test the Understandability of Hybrid Process Models Using DCR Graphs. 30th International Conference on Advanced Information Systems Engineering, EcoKnow Workshop, pages 1-7, 2018.

3. J. Corbin and A. Strauss. Basics of Qualitative Research: Techniques and Procedures for Developing Grounded Theory. SAGE Publications, 2014.

4. J. De Smedt, J. De Weerdt, E. Serral, and J. Vanthienen. Discovering hidden dependencies in constraint-based declarative process models for improving understandability. Information Systems, 74:40-52, may 2018.

5. S. Debois, T. Hildebrandt, M. Marquard, and T. Slaats. Hybrid Process Technologies in the Financial Sector. Proceedings of the Industry Track at the 13th International Conference on Business Process Management 2015, 4:107-119, 2015.

6. M. Dumas, M. La Rosa, J. Mendling, and H. A. Reijers. Fundamentals of Business Process Management. Springer Berlin Heidelberg, Berlin, Heidelberg, 2018.

7. D. Fahland, D. Lübke, J. Mendling, H. Reijers, B. Weber, M. Weidlich, and S. Zugal. Declarative versus Imperative Process Modeling Languages: The Issue of Understandability. In Proceedings of EMMSAD, pages 353-366. Springer, 2009.

8. T. T. Hildebrandt and R. R. Mukkamala. Declarative Event-Based Workflow as Distributed Dynamic Condition Response Graphs. Electronic Proceedings in Theoretical Computer Science, 69:59-73, 2011.

9. K. Holmqvist, M. Nyström, R. Andersson, R. Dewhurst, H. Jarodzka, and J. van de Weijer. Eye Tracking: A comprehensive guide to methods and measures. OUP Oxford, 2011.

10. K. Kluza, K. Kaczor, and G. J. Nalepa. Enriching Business Processes with Rules Using the Oryx BPMN Editor. In Artificial Intelligence and Soft Computing, pages 573-581, Berlin, Heidelberg, 2012. Springer.

11. A. Olsen. The tobii i-vt fixation filter. Tobii Technology, 2012.

12. A. Paivio. Mental Representations: A Dual Coding Approach. OUP, 1990.

13. J. Pinggera, T. Porcham, S. Zugal, and B. Weber. LiProMo-Literate process modeling. CEUR Workshop Proceedings, 855:163-170, 2012.

14. B. Sapkota and M. v. Sinderen. Exploiting rules and processes for increasing flexibility in service composition. In 2010 14th IEEE International Enterprise Distributed Object Computing Conference Workshops, pages 177-185, Oct 2010.

15. W. Wang, M. Indulska, S. Sadiq, and B. Weber. Effect of linked rules on business process model understanding. In Business Process Management, pages 200-215. Springer International Publishing, Cham, 2017.

16. A. J. M. M. Weijters, W. M. P. van der Aalst, and A. K. A. D. Medeiros. Process Mining with the Heuristics Miner Algorithm. TU/e Tech. Report, 166:1-34, 2006.

17. M. Westergaard and T. Slaats. Mixing paradigms for more comprehensible models. pages 283-290, 2013.

18. S. Zugal, C. Haisjackl, J. Pinggera, and B. Weber. Empirical evaluation of test driven modeling. Int. J. Inf. Syst. Model. Des., 4(2):23-43, Apr. 2013.

19. S. Zugal, J. Pinggera, and B. Weber. Creating Declarative Process Models Using Test Driven Modeling Suite. In S. Nurcan, editor, IS Olympics: Information Systems in a Diverse World, pages 16-32. Springer Berlin Heidelberg, 2012.

20. S. Zugal, J. Pinggera, and B. Weber. Toward enhanced life-cycle support for declarative processes. Journal of Software: Evolution and Process, 24(3):285-302, apr 2012 . 